\title{
Riding performance quantification method for motorcycles in terms of collision probability using the logit model
}

\author{
Joohyeong LEE*, Saya KISHINO* and Keisuke SUZUKI* \\ *Division of Intelligent Mechanical Systems Engineering, Kagawa University, Japan \\ 2217-20 Hayashicho, Takamatsu City, Kagawa Prefecture 761-0396, Japan \\ E-mail: suzuki.keisuke@kagawa-u.ac.jp
}

Received: 7 January 2020; Revised: 21 April 2020; Accepted: 30 June 2020

\begin{abstract}
In recent years, many Advanced Driver Assistance Systems (ADAS) have been proposed and introduced under the development of sensing technology and the issue of driving safety. But many kinds of ADASs have a specific threshold to control the alarm or some support. This is decided based on the experimental or mathematical calculations in terms of the optimization of the human-machine interface of each system. But almost all of the systems (especially warning systems) have just a single threshold value to issue the warning, and the driving performance of drivers fluctuating in real time is not considered. In this study, we proposed a quantification method of riding performance and performed the logistic regression analysis for the collision prediction model based on riding performance to optimize the warning threshold of ADAS. For this study, 64 test subjects (Mean age $=22.14$, S.D. $=3.71$ ) participated in the experiments using simulator. Experiments were conducted for three risk events (left-angle collision when a rider was driving on priority road or driving on non-priority road, and right turning collision) and dummy events with the same road environment without risky situations. We proposed a quantification method of riding performance through the total sum of a product of the generalized value of riding behaviours. We also proposed the logit model, which can be constructed in terms of the collision probabilities and riding performance, which is quantified using our proposed method. In the logit model, collision occurrence was used as the dependent variable and riding performance was used as the independent variable for logistic regression analysis to clarify the condition where the probability of collision increases. Finally, we proposed a concept of the setting method of threshold value for the warning timing of ADAS according to the rider's performance level based on collision probabilities during each riding performance.
\end{abstract}

Keywords : Motorcycle, Riding performance, Advanced driver assistance system (ADAS), Advanced rider assistance system (ARAS), Logistic regression analysis, Evaluation method, Collision probability

\section{Introduction}

\subsection{Motivation for the study}

Various kinds of studies for traffic safety are being carried out to evaluate the driver's driving performance in risky situations or usual driving. In most studies, braking or steering reaction time during driving is regarded as the driving performance (Consiglio, W. et al., 2003; Philip, P. et al., 2005; Beede, K. E., and Kass, S. J., 2006) or the driving behaviours of the drivers are evaluated as each variable (Van Der Hulst, M. et al., 2001; Charlton, S. G., 2009). Of course, such steering reaction time or brake reaction time is the most important driving behaviour during the driving operation, and that is the most important variable affecting traffic accidents (Green, M., 2010), but other driving behaviour such as deceleration and misjudgement are also crucial variables that affect traffic accidents. Moreover, riding skills such as dynamic balance and control of the motorcycle, judgment of safety margin to obstacles or dangerous vehicles, appropriate input to initiate swerve, reaction time, etc. are evaluated to understand which riding skills are important for variable task scenarios as a riding performance (Creaser, J. I. et al., 2009). The eye-movement 
was investigated to confirm the risky behaviour as a riding performance by comparing novice riders and experienced riders (Di Stasi, L. L. et al., 2011). Investigating the important driving behaviour which has a direct impact on the possibility of traffic accidents and expressing the driving performance as a single index enables us to compare driving performance of drivers intuitively, and the quantified driving performance can be used as an evaluation index for the new Advanced Driver Assistance System (ADAS). Most of the research on driving performance have been carried out for the development of four-wheeled vehicles. But the riding performance of two-wheeled vehicle riders, who require higher driving performance during driving than four-wheeled vehicle drivers, has not been studied in detail. Moreover, the injury rate of the two-wheeled vehicle riders is 3 to 8 times more frequent than four-wheeled vehicle drivers when they are novice two-wheeled vehicle riders, and the actual driving experience is required to reduce accidents (Mayberry, J. C. et al., 2007). ADAS for four-wheel vehicles is an active research item, and this has evolved in various directions due to the development of technologies such as Vehicle to Everything (V2X), cameras, radar, and sensors (Okuda, R. et al., 2014). In the case of two-wheeled vehicles, Riding Simulator (RS) has been developed to train novice riders and analyse riding safety (Nehaoua L. et al., 2007; Cossalter, V. et al., 2008). However, many RS studies focus on controlling the dynamics of vehicles. Since 2008, Advanced Rider Assistance System (ARAS) and On-Bike Information System (OBIS) have been actively studied by the SAFERIDER project (Bekiaris, E. D. et al., 2009) for two-wheeled vehicle riders. The SAFERIDER team studied five key ARAS functions: speed alert, curve warning, frontal collision warning, intersection support, and lane change support. Many kinds of Collision Avoidance/Collision Warning (CA/CW) algorithms and systems have been proposed since the early 1990s mostly by industrial researchers and algorithm developers (Doi, A. et al., 1994; Lee, K., and Peng, H., 2005). Among them, CA/CW algorithms, Forward Collision Warning (FCW) systems algorithms target major rear-end crashes with other vehicles which have a risk of collision that accounts for $35 \%$ of all accidents. FCW is based on the CA algorithms, which can be divided into two categories: Perceptual-Based Warning Algorithms and Kinematic-Based Warning Algorithms (Wang, X. et al., 2016) as Table 1 . The perceptual-based warning algorithms trigger a collision alarm based on the time with the other vehicle that may collide with the ego vehicle if driving continuously. For example, Time to Collision (TTC) is used as a threshold value. The advantage of this perceptual-based algorithms is that it calculates relative velocity, and velocity of the ego vehicle and it is meaning no necessary to calculate other vehicle's information such as the deceleration in real time. However, its disadvantage is that human perceptual ability and driving ability are not included, so there is a possibility that accuracy may be lower in various risk situations. The kinematic-based warning algorithms trigger a collision alarm based on the theoretical distance to stop safely. The alarm threshold is calculated based on the relative distance between the vehicles, deceleration of the ego vehicle and other vehicle, the driver's reaction time, etc. However, this method requires many sensors, and its computation becomes complicated, and calculation can be made with various assumptions.

Table 1 Comparison equations of previous Forward Collision Warning (FCW) algorithms.

\begin{tabular}{|c|c|c|c|}
\hline $\begin{array}{c}\text { Type of } \\
\text { Algorithm }\end{array}$ & $\begin{array}{l}\text { Name of } \\
\text { Algorithm }\end{array}$ & Equation & Reference \\
\hline \multirow{3}{*}{$\begin{array}{l}\text { Perceptual-Based } \\
\text { Warning } \\
\text { Algorithms }\end{array}$} & $\begin{array}{l}\text { Honda's TTC } \\
\text { algorithm }\end{array}$ & $\mathrm{R}_{\text {warning }}=f\left(V_{\text {rel }}\right)=2.2 V_{\text {rel }}+6.2$ & $\begin{array}{c}\text { Fujita, Y., } \\
\text { Akuzawa, K. } \\
\text { and Sato, M. } \\
\text { (1995) }\end{array}$ \\
\hline & $\begin{array}{l}\text { Hirst and } \\
\text { Graham' TTC } \\
\text { algorithm }\end{array}$ & $R_{\text {warning }}=f\left(V_{\text {rel }}, V_{S V}\right)=3 V_{\text {rel }}+0.4905 V_{S V}$ & $\begin{array}{l}\text { Hirst, S. and } \\
\text { Graham, R. } \\
\quad(1997)\end{array}$ \\
\hline & $\begin{array}{l}\text { Bella and Russo } \\
\text { algorithm }\end{array}$ & $R_{\text {warning }}=1.25 V_{\text {rel }}+1.55 V_{S V}$ & $\begin{array}{l}\text { Bella, F. and } \\
\text { Russo, R. } \\
\text { (2011) }\end{array}$ \\
\hline \multirow{2}{*}{$\begin{array}{l}\text { Kinematic-Based } \\
\text { Warning } \\
\text { Algorithms }\end{array}$} & $\begin{array}{l}\text { Stop Distance } \\
\text { Algorithm }\end{array}$ & $R_{\text {warning }}=V_{S V} R T+\frac{V_{S V}^{2}}{2 a_{S V}}-\frac{V_{L V}^{2}}{2 a_{L V}}$ & $\begin{array}{l}\text { Wilson, T. B. et } \\
\text { al., (1997) }\end{array}$ \\
\hline & $\begin{array}{l}\text { Mazda's } \\
\text { algorithm }\end{array}$ & $\begin{array}{c}R_{\text {warning }}=V_{S V} \tau_{1}+V_{\text {rel }} \tau_{2}+\frac{V_{S V}^{2}}{2 a_{S V}}-\frac{V_{L V}^{2}}{2 a_{L V}} \\
+R_{\text {min }}\end{array}$ & $\begin{array}{l}\text { Doi, A. et al., } \\
\text { (1994) }\end{array}$ \\
\hline
\end{tabular}


Here, for the perceptual-based warning algorithms, $\mathrm{R}_{\text {warning }}$ is Warning Onset Range (WOR) $(\mathrm{m}), f\left(v_{\text {rel }}\right)$ is relative velocity $(\mathrm{m} / \mathrm{s})$ between the two vehicles, $V_{S V}$ is velocity of the subject vehicle. And for the kinematic-based warning algorithms, $V_{L V}$ is velocity of the leading vehicle, $R T$ is reaction time, $a_{S V}$ is deceleration of the subject vehicle, $a_{L V}$ is deceleration of the leading vehicle, $\tau_{1}$ is the system delay, $\tau_{2}$ is the driver delay, and $R_{\min }$ is the minimum safety range. And many warning algorithms were evaluated by K. Lee and H. Peng (2005) such as Mazda, Honda (w, a), Jaguar, and JHU-APL algorithm using the Intelligent Cruise Control Field Operational Test (ICCFOT) database. These algorithms can calculate a threshold distance by the motion of vehicles and driving behaviour such as ego vehicle velocity, other vehicle velocity, acceleration or deceleration of vehicles, and human delays. They concluded that the JHU-APL logic has the best performance index in the other three algorithms, but its performance is still less than satisfactory. And they confirmed that the extremely simple TTI index has higher accuracy than all evaluated algorithms (Lee, K., and H. Peng., 2005). These algorithms or systems are no longer prototypes and are not systems of the future. These systems have already been introduced in many vehicles and efforts need to be made to increase accuracy for improving driver safety. In addition, the threshold of many conventional ADAS such as systems using perceptual-based warning algorithms are decided as a single value calculated considering the vehicle dynamics (Liu, H. et al., 2017). The kinematic-based warning algorithms have a variable threshold timing for the collision warning, but these algorithms also must assume the driver's reaction time and ego vehicle acceleration.

These system algorithms still have a problem that it can be too late/unreliable for some drivers, too early/annoying for some drivers (Vahidi, A., and Eskandarian, A., 2003). We think that this is because the trigger threshold is calculated without considering/assuming the driving performance of each driver. In our previous study (Lee, J. et. al., 2018), we simulated and quantified riding behaviour when using and not using ADAS for two-wheeled vehicles. As a result, there was no significant difference in riding behaviour among the test subjects when ADAS was not used, but it was confirmed that riding behaviour changed according to the rider's riding style when using ADAS. Unlike the safety system of the four-wheeled vehicle, for the design of safety systems of two-wheeled vehicle, the riding style and riding performance should be applied as variables in the safety system algorithm. If the rider's characteristics are applied to the control algorithm of safety system, the accidents caused by collisions can be significantly reduced, and the usefulness of the safety system can also be increased. Therefore, in this study, we proposed a method to quantify the rider's riding behaviour as one variable as riding performance, and we also proposed a model to predict riding performance in real time. Moreover, we will discuss a concept to apply predicted riding performance to the control algorithm of ADAS.

\subsection{Purpose of the study}

To quantify the riding performance of the two-wheeled vehicles, which have more critical impact for collision accidents than driving performance of a four-wheeled vehicles, we proposed a quantification method of the riding performance of two-wheeled vehicle riders. The aim is to estimate the riding performance value as a single indicator by investigating the riding behaviour to prevent the collision. For estimating the riding performance, the investigated riding behaviour was normalized, and additional scores which represent the importance of the impact of collision occurrence were added. We also proposed a mathematical model that reflects the riding performance of the individual riders using quantified riding performance for determining the threshold to control ADAS. This model was constructed using logistic regression analysis to predict the collision probability of each riding performance amount. In this study, we have the following two purpose:

\section{(1) Propose a riding performance quantification method}

This aims to investigate the rider's riding behaviours in risky situations and to quantify the impact on the collision probability. We also proposed a method to explain a single indicator as a riding performance of two-wheeled vehicle riders. We investigated the riding behaviour of the participants in the risky situation through experiments using a riding simulator and proposed a quantification method based on such riding behaviour, which affects collision. It is expected that this proposed quantification method can be used as an evaluation method of ADAS in terms of collision mitigation and it is expected to propose new functions of ADAS considering the performance of the two-wheeled vehicle riders.

(2) Propose a mathematical model to predict the collision probability based on the riding performance of two-wheeled riders using logistic regression analysis 
This aims to model the two-wheeled vehicle rider's riding performance and collision probability to monitor the preparation behaviour for collision avoidance in risky situations. Therefore, we used logistic regression analysis with the defined riding performance and collision probability to investigate the possibility of collision during each riding performance. Also, we proposed a concept of the threshold setting method to consider the riding performance level.

\section{Quantification method of riding performance based on riding behaviour of two-wheeled vehicle riders in risky situations}

\subsection{Riding simulator for investigating riding behaviour of two-wheeled vehicle riders}

In this study, we quantified the riding performance of two-wheeled vehicle riders to construct a mathematical model which shows the relationship between riding performance and collision probability when riders are in risky situations. For investigating the riding behaviour and collision occurrence of the riders in risky situations, the riding simulator experiments were conducted to consider variable conditions, scenario manipulation, session time, participation of multiple subjects, and the safety of test subjects during risky situations. Figure 1 shows the riding simulator (RS) constructed for the experiments to investigate riding behaviour. Our RS has a main-PC for calculating the vehicle dynamics, sub-PC for calculating and transmission of graphics, head-mounted display (HMD) for projecting the road environment, the electrical fan for increasing the reality of driving velocity, and actual motorcycle frame that can investigate the riding behaviour by the steering angle, opening level of the accelerator, and pressure level of brake handle.

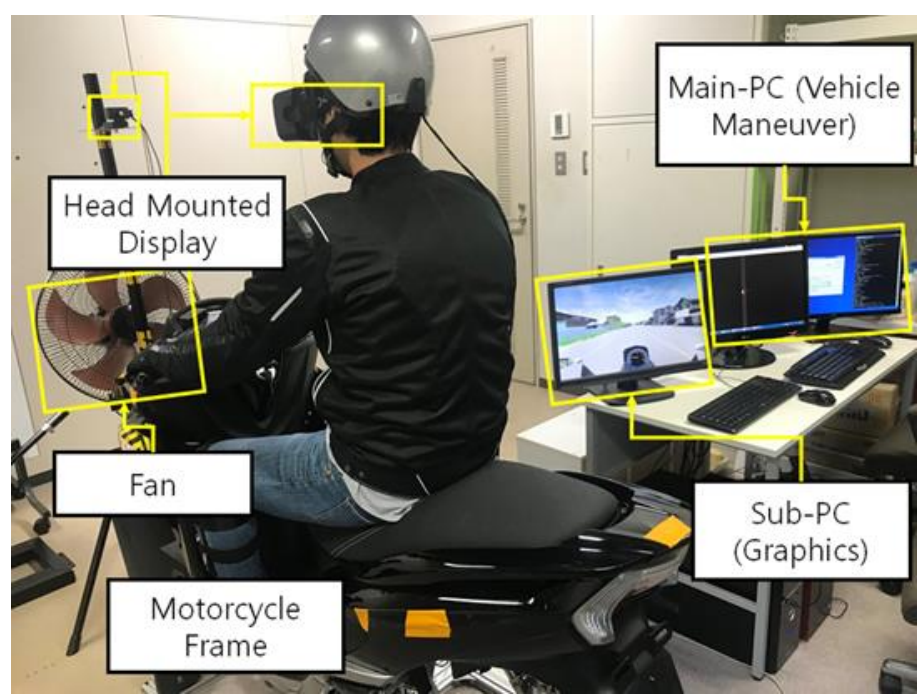

Fig. 1 Riding simulator to investigate riding behaviour for quantification of riding performance of two-wheeled vehicle riders and to construct a logit model to predict collision probability using riding performance.

The HMD was used to reproduce "turning the head to check the surrounding situations" that driving characteristic of two-wheeled vehicle riders and the HMD used Oculus' Rift development kit 2. For the simulator frame, Yamaha' Tricity 125 frame was used to enhance the realism of simulation. However, since it is a simulator using HMD, there is a risk of test subjects falling from the riding simulator during the experiment, so the simulator frame is constructed as a static type without dynamic motion systems. The electric air fan was also used to enhance the realism of the simulation, and the wind velocity was controlled according to the speed of the own vehicle in the simulation. The air fan used YSD-455Y (K) manufactured by Yuasa Primus, which is equipped with a 40W DC motor, to control the wind velocity. The wind velocity was controlled using an Arduino connected with the simulator. We simulated the experimental conditions and calculated motorcycle dynamics using MatLab R2013a-Simulink and linked it to Car-Sim 8.2.2. We used DS-nano supported by Misaki-design as platform software for driving simulation.

\subsection{Experiment design}

This study was carried out to investigate the riding behaviour of two-wheeled vehicle rider in risky situations and to specify the degree of preparation for the collision accidents. We investigated three riding behaviours which are related to collisions such as brake reaction time (BRT), average deceleration (AD), and time to collision (TTC) when 
the risky situations are encountered. Here, BRT means the time at which the drivers are required to start the braking operation after detecting risky situations or vehicles, $\mathrm{AD}$ means the average value of deceleration which is from the start of brake operation until the vehicle stops completely, and TTC indicates the remaining time to collision between the ego vehicle driver and other vehicle.

The riding simulator experiments were conducted with three different scenarios for the accident pattern of the risky events, and dummy scenarios which have no risk events in the same road environment were also conducted to increase the reality of the experiments. The risk events were designed based on the frequent traffic accidents occurring at the intersections in Japan, the three risk events consisted of the two types of left-angle collision when a rider is driving on priority roads or driving on non-priority roads, and one type of right turning collision at the intersection as shown in Fig. 2. The left-angle collision is the accident pattern between a two-wheeled vehicle rider driving straight and a car emerging from a side road at the intersection. In this study, we defined the left-angle collision known as T-bone collision or right-angle collision in other countries (because the driving lane is the opposite side to other countries in Japan), and this collision pattern is one of the most frequent accident patterns in Japan according to traffic statistics report of ITARDA. Right turning collision occurs between a motorcycle rider driving straight on the road and a car turning to its right from the opposite lane at the intersection, and this collision pattern is the second most frequent accident pattern in Japan (Traffic statistics report of ITARDA).

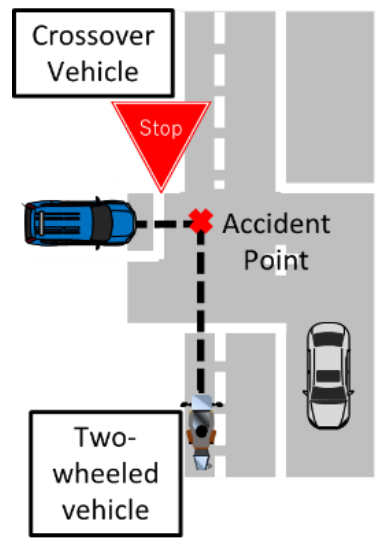

(a) Scenario \#1

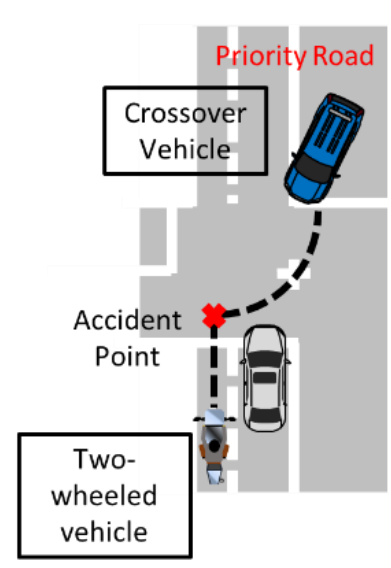

(b) Scenario \#2

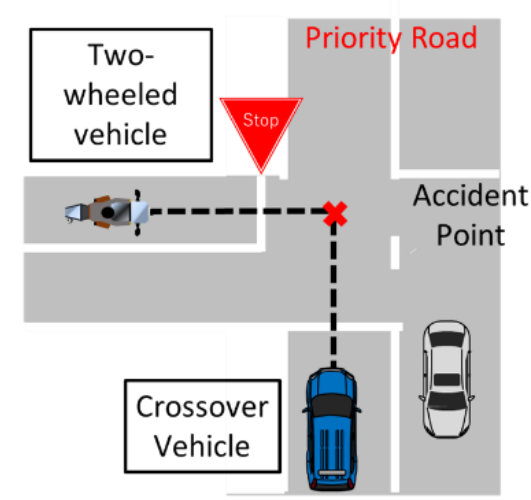

(c) Scenario \#3

Fig. 2 Riding simulator experiment scenarios for investigating riding behaviour in risky situations

\subsection{Experiment procedure}

We performed the riding simulator experiments to quantify the riding performance when riders are in risky situations with the 64 test subjects (Mean age $=22.14$, S.D. $=3.71$ ), who have a two-wheeled vehicle driving license and who are not novice riders. In order to reduce the learning effect as much as possible, the test subjects were divided into two groups based on scenarios: the subject's own vehicle traveling on the priority road and the subject's vehicle driving on the non-priority road. The test subjects selected were, 30 persons (Means age $=22.1$, S.D. $=1.49$ ) for scenario \#1 and \#2, and 34 persons (Mean age = 22.18, S.D. = 4.93) for scenario \#3 as shown in Table 2. During the riding simulator experiments, the test subjects were required to drive to the destination and perform actions for collision avoidance in the case of risky situations. We required test subjects to perform the Uchida-Kraepelin test (Kuraishi, S., 2000; Suzuki, K. et al., 2016) as a sub-task when they were driving at the same time to reproduce the mental workload during the actual driving. To reduce the learning effect as much as possible and limit the other variables to be investigated, the experimental scenarios were randomized for each test subject. We gave the test subjects enough time to relax during the experiment and when they want. We gave the test subjects enough rest time after the experiment.

Before starting the riding simulator experiment, we explained the experiment procedure thoroughly and obtained the preliminary informed consent. All test subjects joined the experiment after signing an informed consent, the content was reviewed and approved by the Experiment Ethics Committee of Kagawa University before the experiments. 
Table 2 Conditions of riding simulator experiment scenarios to investigate riding behaviours in risky situations.

\begin{tabular}{l|l|l|l}
\hline \hline No. & Experiment Scenarios & Groups & Test Subjects \\
\hline$\# 1$ & Left-angle collision (Priority road) & $\# 1$ & 30 Persons \\
\hline$\# 2$ & Right-turn Collision (Priority road) & & \\
\hline$\# 3$ & Left-angle collision (Non-Priority road) & $\# 2$ & 34 Persons \\
\hline
\end{tabular}

\subsection{Quantification of riding performance based on riding behaviour}

The riding performance was quantified as a summation of the product of the generalized value based on each riding behaviour (Parameters) which related to the collision accident and weight value which represent the effect on the collision. The overall riding performance was calculated using the following formula:

$$
\begin{aligned}
& P_{\text {Overall }}=\left(a_{1} * P_{1}\right)+\left(a_{2} * P_{2}\right)+\cdots+\left(a_{n} * P_{n}\right) \\
& P_{\text {Overall }}=\sum_{i=1}^{n}\left(a_{i} * P_{i}\right)
\end{aligned}
$$

Here, $P_{\text {Overall }}$ is the overall riding performance, $a_{i}$ is the weight value of each performance and $P_{i}$ is the generalized riding performance for each parameter. Where the $P_{i}$ (Performance of parameter) is:

if the parameter has a positive correlation with collision:

$$
P_{i}=\frac{\text { Parameter }_{\text {max }}-\text { Parameter }_{i}}{\text { Parameter }_{w}}
$$

if the parameter has a negative correlation with collision:

$$
P_{i}=\frac{\text { Parameter }_{i}-\text { Parameter }_{\min }}{\text { Parameter }_{w}}
$$

Here, Parameter $_{\text {max }}$ is the maximum value of each parameter, Parameter $_{\text {min }}$ is the minimum value of each parameter, and Parameter $_{w}$ is the value of Parameter $_{\text {max }}$ minus Parameter $_{\text {min }}$. Through this, we generalize each parameter from 0 to 1 . Also, all parameters were defined as the performance is increased from 0 to 1 , even the parameter has a negative correlation with collision occurrence. The correlation analysis of each riding behaviour (Parameters) and collision were tabulated in Table 3 to decide the weight value for representing how much is related to the collision as a value. The reason for investigating the correlation between the frequency of collision and each parameter is that a good riding performance is "driving without collision" rather than "driving well". From the correlation analysis, the causality between each parameter and the occurrence of the collision was quantified numerically, and the quantified causality was used to determine the weight value. Correlation analysis can find that each parameter has a positive or negative relationship with collision, and decide the weight value, the Pearson correlation was used proportionally. We decided the summation of all weight value is 1 for representing the overall performance from 0 to 1 and the decided weight values of each riding behaviour are, 0.4 for BRT, 0.1 for AD, and 0.5 for $\mathrm{TTC}_{\min }$ as shown in Table 4. Each weight value was applied proportionally to the Pearson's correlation coefficient, and the sum of all weight value was set to 1 . 
Table 3 Correlation between riding behaviour and collision occurrence.

\begin{tabular}{l|l|l|l|l}
\hline \hline & & Brake Reaction Time & Average Deceleration & $\mathrm{TTC}_{\min }$ \\
\hline \multirow{4}{*}{ Collision } & $\begin{array}{l}\text { Pearson } \\
\text { Correlation }\end{array}$ & 0.313 & -0.070 & -0.476 \\
\cline { 2 - 5 } & $\begin{array}{l}\text { Sig. } \\
(2 \text {-tailed })\end{array}$ & 0.000 & 0.116 & 0.000 \\
\cline { 2 - 5 } & $\mathrm{N}$ & 502 & 506 & 507 \\
\hline
\end{tabular}

(Avoidance: 0, Collision: 1)

From this, we can understand the $P_{B R T}$ will increase when BRT value is low (when start braking operation is fast), $P_{A D}$ will increase when $\mathrm{AD}$ value is high (when deceleration is fast), and $P_{T T C}$ will increase when $\mathrm{TTC}_{\min }$ value is high (when stopped far from the other vehicle). Also, we can understand the extent of the effect on the collision occurrence from each parameter performance, and from the overall riding performance, which is represented from 0 to 1. Of course, each parameter performance and overall performance can represent the other value of width.

Table 4 Weight value of each parameter to quantify riding performance.

\begin{tabular}{l|l|l|l}
\hline \hline & Brake Reaction Time & Average Deceleration & $\mathrm{TTC}_{\min }$ \\
\hline Weight of Performance & 0.4 & 0.1 & 0.5 \\
\hline Pearson Correlation & 0.313 & -0.070 & -0.476 \\
\hline
\end{tabular}

\section{Modelling of riding performance and collision probability using logistic regression analysis}

\subsection{Modelling of riding performance and collision probability for all of riding characteristic}

Figure 3 illustrates the histogram of quantified riding performance sorted by the occurrence of collision in the riding simulator experiments. The occurrence of collision started when the riding performance of two-wheeled vehicle riders is under around 0.3 points. However, avoidance of risky situation was also confirmed at the same level of riding performance. So, we must understand the collision probability based on each riding performance levels. If we can understand how much value of riding performance is good or bad for collision avoidance, we may apply our model to ADAS for setting the threshold such as alarm timing, monitoring of performance or arousal level, and many concepts with considering the individual riding performance.

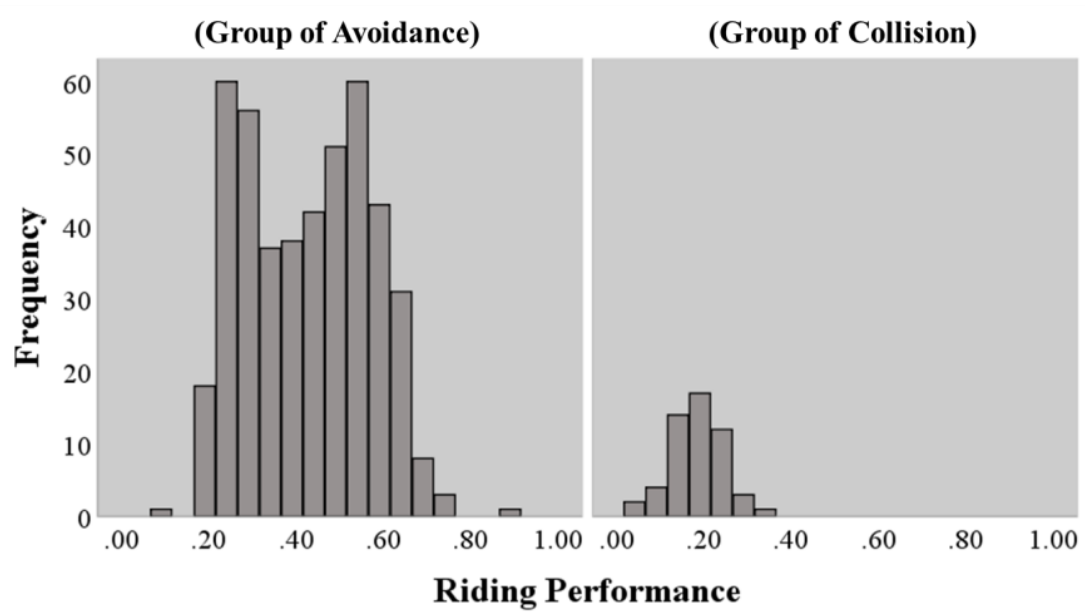

Fig. 3 Histograms of quantified riding performance of two-wheeled vehicle riders sorted by collision occurrence in riding simulator experiments. 
In order to create a model that can predict collisions with riding performance, modeling was performed using statistical regression analysis. Prior to modeling, a regression model was selected. In general, logistic analysis is used for regression models with continuous variables as independent variables and nominal variables as dependent variables. Logistic regression analysis was selected to modeling because the independent variable in our model is the continuous variable (Riding performance), and the dependent variable is a nominal variable (Collision occurrence). The logistic regression analysis was performed to understand how much value of riding performance is good or bad in terms of vehicle safety. The concept of logistic regression analysis was proposed by D.R. Cox (1958) to predict the likelihood of an incident using a linear combination of independent variables. Generally, the logistic regression analysis was used to predict the event (event occurs or does not occur) mainly in the field of medical and natural science research as a classification method. The dependent variable of the logistic regression analysis is a binomial value such as collision occurs, or collision does not occur unlike the linear regression analysis using continuous variables as independent variables. Typically, the logit model (or logistic model), which is constructed by logistic regression analysis, is used to predict the event occurrence as a classifier. However, in this study, we used the logit model to predict the probability of collision of each riding performance level. In our model, the collision occurrence was used as the dependent variable (0: avoidance, 1: collision) and riding performance was used as the independent variable for logistic regression analysis to find the point where the probability of collision increases. Through the logit model based on riding performance and collision rate, we can also decide the threshold for the onset control timing ADAS.

The result of logistic regression analysis to predict collision occurrence based on riding performance is shown in Table 5, and Eq. (5) is the constructed logit model to predict the collision probability using the quantified riding performance. In the model, the regression coefficient of the riding performance was -33.722 ( $p<0.001)$, and the intercept was 6.534 (p < 0.001). We confirmed the model which has as Akaike Information Criterion (AIC) 156.1, and McFadden $\mathrm{R}^{2}$ was 0.632 . The AIC was proposed by Akaike (1971) to compare the relative quality of statistical models. Typically, AIC was used to compare the goodness of fit of models, and lower value of AIC indicates a good model. When making measurement data into a statistical model, AIC is used to check the balance of the complexity and the Goodness of Fit (GOF) of the statistical model. In this section, AIC was just applied because there are no other models to compare. The $\mathrm{R}^{2}$ is used to verify the fitness of the linear regression model, but the fitness of the model cannot be verified with the $\mathrm{R}^{2}$ since the logistic regression analysis uses the Generalized Linear Model (GLM). There are many ways to verify the fitness of a logistic model such as the Cox-Snell $R^{2}$, Nagelkerke $R^{2}$, and McFadden $R^{2}$. The McFadden $\mathrm{R}^{2}$ has been tested to confirm the fitness of our model. McFadden $\mathrm{R}^{2}$ was proposed by McFadden (1974) to verify model fitness based on the maximized likelihood value from the current fit model and value corresponding to the null model. In general, the good value of McFadden $\mathrm{R}^{2}$ is known to be between 0.2 and 0.6 , and the McFadden $\mathrm{R}^{2}$ of our model is 0.632 , so we believe our model is reliable.

Table 5 Result of logistic regression analysis based on riding performance and collision.

\begin{tabular}{l|l|l|l|l}
\hline \hline & Estimate & Std. Error & z value & $\operatorname{Pr}(>|\mathrm{z}|)$ \\
\hline $\begin{array}{l}\text { Intercept } \\
\text { Riding } \\
\text { Performance }\end{array}$ & 6.534 & 1.088 & 6.007 & $1.89 \mathrm{e}-09 * * *$ \\
\hline
\end{tabular}

AIC: 156.1

McFadden $\mathrm{R}^{2}: 0.632$

$$
\text { Collision Probability }=\frac{1}{1+e^{-\left(\left(-33.722 \times P_{\text {Overall })+6.534)}\right.\right.}}
$$

Figure 4 shows the prediction of collision probability based on riding performance using our logit model (Fig. 4 (a)), and Receiver Operating Characteristics Curve (ROC) which represents the accuracy of our model (Fig. 4 (b)). The model prediction was performed from the data which was investigated from riding simulator experiments, and we 
confirmed and quantified the collision probability of each riding performance level. The collision probability begins to increase when riding performance is under 0.34 . And when riding performance is under 0.19 , the collision probability begins to increase over $50 \%$ in our model. The ROC has two axes, the x-axis represents False Positive Rate (FPR) or 1 - Specificity, which is the rate at which the classifier predicts negative when the original data is positive, and the $y$-axis represents True Negative Rate or Sensitivity (TNR) which is the rate at which the classifier predicts negative when original data is negative. Since the ROC is a graph, it is difficult to quantify the model accuracy, so the Area Under Curve (AUC) is used to quantify the accuracy of the model. The AUC value is the area under the line of the ROC graph, the maximum value of AUC is 1 , and it means perfect classifier. In general, a higher value of AUC indicates a higher accuracy, but when the value is closer to 0.5 , it is not to be classified and is closer to random. AUC of our model is around 0.96 of, and it seems to have quite a high accuracy.

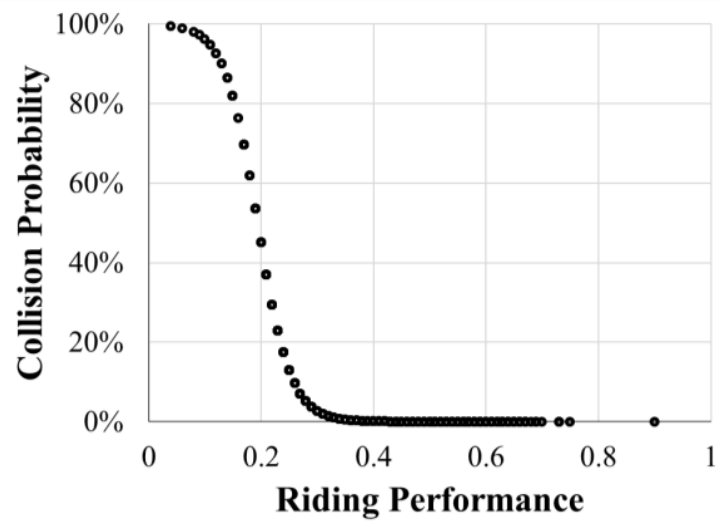

(a)

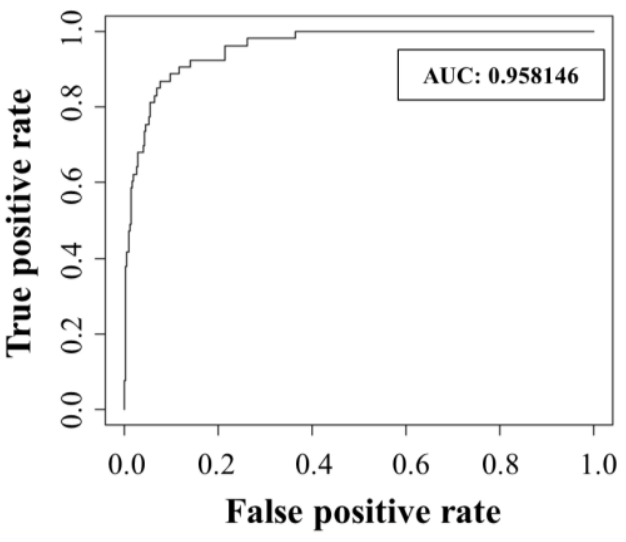

(b)

Fig. 4 Collision probability prediction based on riding performance using logistic regression (a) and ROC graph of logit model to predict collision based on riding performance $(b)$.

\subsection{Modelling of riding performance and collision probability according to the rider's riding style based on Riding Style Questionnaire (RSQ)}

We performed a hierarchical cluster analysis based on the Riding Style Questionnaire (RSQ) to investigate the riding performance depending on the riding style of the riders. The RSQ is the modified version to investigate the riding style for two-wheeled riders based on Driving Style Questionnaire (DSQ) reported by Ishibashi, M. (2008) to investigate the driving style of four-wheeled drivers. The RSQ consists of 18 questions as four-scales to quantify the riding style of test subjects. The riding style can be classified according to eight characteristics such as confidence or passivity to riding ability, patient or impatient riding tendency, anxiousness or unstable driving, methodical riding tendency, and how they think the car as a status symbol. The RSQ is a questionnaire about riding style, not about riding behaviour, so clustering analysis is categorized on how they think about riding rather than riding behaviour. As a result of clustering analysis, we can divide the riding style into two groups as group A with 38 test subjects who have the tendency of relatively high anxiousness and low confidence for riding, and group B with 26 test subjects who have the tendency of low anxiousness and high confidence for riding as shown in Fig. 5 (a). In Figure 5 (b), comparison of riding performance for group A and group B is shown. The riding performance of group A (Mean performance $=0.406$, S.D. $=0.148)$ is slightly higher than the riding performance of group B (Mean performance $=0.381$, S.D. $=0.164)$ but the result of $t$-test did not indicate a significant difference $(t=1.710, p=0.088)$. We had considered that the riding performance of group B is higher than group A, but the result was different. The riding performance was quantified as preparation for collision avoidance. Therefore, the riding performance of group A, which tends to have high anxiousness and low confidence for riding, was higher than group B. We think the tendency of low confidence and high anxiousness for riding has enhanced the riding performance for driving safety. 


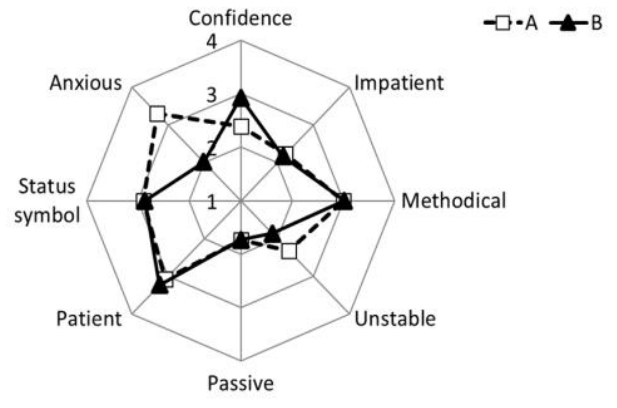

(a)

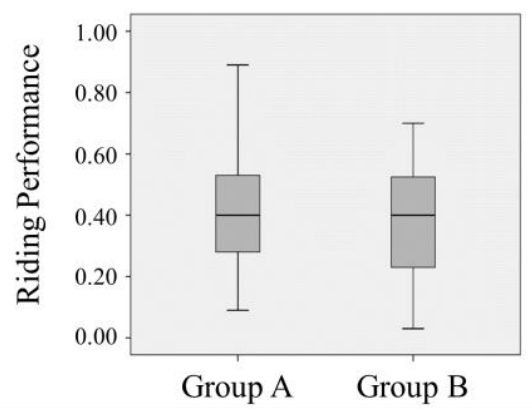

(b)

Fig. 5 Clustered two riding styles of test subjects by riding style questionnaire (a) and quantified riding performance sorted by cluster group based on riding style (b).

The logistic regression analysis was performed for both groups A and B to quantify the collision probability at each riding performance level and to compare the difference with different rider characteristics. Table 6 is a result of logistic regression analysis for group A and B. The regression coefficient of the riding performance was -30.287 (p < 0.001$)$ for group A and -49.638 ( $p<0.001$ ) for group B, the intercept was $5.877(\mathrm{p}<0.001)$ for group B and 9.225 (p < 0.001$)$ for group B. We confirmed that the model of group B has better goodness of fit according to AIC of a model which was smaller than the model for group A (Group A: 95.184, Group B: 56.912). We think that this is because the collision probability of group B (Collision probability $=13.94 \%$ ) is higher than group A (Collision probability $=8.16 \%$ ), so the situation of collision is better represented when the model was constructed. It was also confirmed that McFadden $\mathrm{R}^{2}$, and AUC of each model for group B has higher accuracy than the model for group A. The McFadden $\mathrm{R}^{2}$ is 0.522 for model of group A and 0.767 for model of group B, AUC is 0.944 for model of group A and 0.978 for model of group B. However, we think that both models for group A and B have a high accuracy in predicting the collision probability based on riding performance. Figure 6 illustrates the prediction of collision probability based on riding performance using logit model for groups A and B. The predicted collision probability of group A began to increase gradually when the riding performance was less than 0.368 , and when the riding performance was less than 0.194 , the collision probability exceeded $50 \%$. However, the predicted collision probability of group B began to increase rapidly when the riding performance was less than 0.292 , and the collision probability exceeded $50 \%$ when the riding performance was less than 0.186 . This means the collision probability rapidly increases when riding performance was less than a certain level of riding performance, riders with the characteristic of group B. Therefore, we have confirmed the difference in riding performance (in terms of vehicle safety) according to the rider's riding style. Moreover, we have confirmed that there is little difference in the models to predict the collision based on riding performance. Therefore, these models need to be customized to their characteristics, and we think that this method can improve the accuracy of information presentation timing of ADAS based on considering the riding performance.

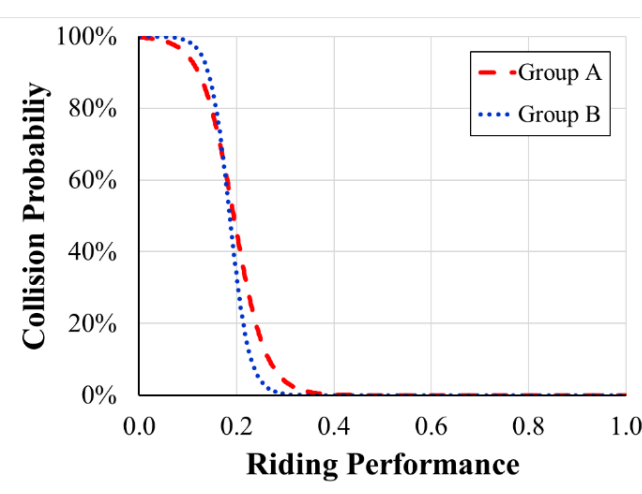

(a)

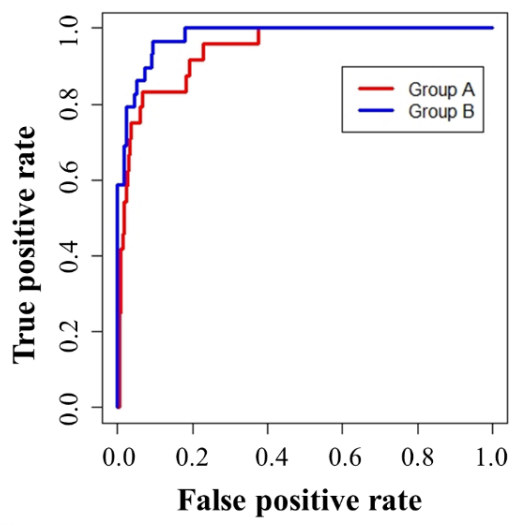

(b)

Fig. 6 Collision probability prediction of group A and B based on riding performance using logistic regression (a) and ROC graph of logit model of group A and B to predict collision based on riding performance (b). 
Lee, Kishino and Suzuki, Mechanical Engineering Journal, Vol.7, No.4 (2020)

Table 6 Result of logistic regression analysis for group A and B based on riding performance and collision.

\begin{tabular}{|c|c|c|c|c|c|c|c|c|}
\hline \multicolumn{2}{|c|}{ Group } & Estimate & $\begin{array}{l}\text { Std. } \\
\text { Error }\end{array}$ & $\mathrm{z}$ value & $\operatorname{Pr}(>|z|)$ & AIC & McFadden $\mathrm{R}^{2}$ & AUC \\
\hline \multirow[b]{2}{*}{ A } & Intercept & 5.877 & 1.474 & 3.987 & $\begin{array}{c}6.69 \mathrm{e}-05 \\
* * *\end{array}$ & \multirow[b]{2}{*}{95.184} & \multirow[b]{2}{*}{0.522} & \multirow[b]{2}{*}{0.944} \\
\hline & $\begin{array}{c}\text { Riding } \\
\text { Performance }\end{array}$ & -30.287 & 6.244 & -4.851 & $\begin{array}{c}1.23 \mathrm{e}-06 \\
* * *\end{array}$ & & & \\
\hline \multirow{2}{*}{ B } & Intercept & 9.225 & 2.165 & 4.260 & $\begin{array}{c}2.04 \mathrm{e}-05 \\
* * *\end{array}$ & \multirow{2}{*}{56.912} & \multirow{2}{*}{0.767} & \multirow{2}{*}{0.978} \\
\hline & $\begin{array}{c}\text { Riding } \\
\text { Performance }\end{array}$ & -49.638 & 10.890 & -4.558 & $\begin{array}{c}5.16 \mathrm{e}-06 \\
* * *\end{array}$ & & & \\
\hline
\end{tabular}

\section{Limitations and Discussion}

\subsection{Quantification of riding performance based on riding behaviour}

In this study, the riding simulator experiments were performed using the static type driving simulator to simulate straight course scenarios. Since this experiment was performed with a riding simulator using HMD, the experiment was conducted with a static type of driving simulator for the safety of the test subjects. All subjects received adequate instructions before the experiment. During the experiment, all test subjects wore safety gear that is used during actual driving. We used the HMD to increase the driving reality, the blowing of wind was correlated to the vehicle speed, and the sub-task was set up to increase the mental workload to reproduce the actual operation during the riding simulator experiments. As mentioned in section 2.1, the riding simulator was constructed as a static type simulator for the test subjects' safety, since they can not see the surrounded environments. To increase the reality of the simulator, the maneuver of motorcycle and vehicles in simulation scenes was controlled by CarSim software. However, the motorcycle is a vehicle that has dynamic movements. Therefore, investigated riding behaviours using static type simulator may differ from the riding behaviours using dynamic type simulator. Many studies have discussed whether such a safety system would be effective in a real driving environment. Hoe C. Lee. et al., (2003) conducted an Field Operational Test (FOT) and the simulator experiment to evaluate the driving performance of elderly drivers. The experiment was conducted with 129 test subjects of elderly drivers and the index to quantify the driving performance was suggested. As a result, a positive correlation was confirmed between the riding performance at the FOT and the simulator experiment. Also, they mentioned the simulator experiment can be a more economical method to test the driving performance than the FOT. Mayhew, D. R. et al., (2011) compared various driving behaviours in FOT and simulator experiments to quantify the driving performance of the novice drivers. They also confirmed that each driving behaviours have a positive correlation at the FOT and the simulator experiment. From these studies, validating a safety system in a simulator environment may be uncertain or less accurate compared to the FOT, but it is sufficient to predict the effectiveness of safety systems. In addition, verification of safety systems in a simulator environment is also being verified in the field of reliability engineering. Suzuki, K. et al., (2016) proposed an evaluation method of safety systems using experiments with the simulator environment and a time series simulation based on reliability engineering. They proposed a model to obtain reliable data by reducing statistical errors from experiments. The suggested model for the safety system has also been verified in the simulation environment, but its effectiveness will also linearly proportional to the riding behaviours in the actual driving situations. However, the experiments at the laboratory are not the same as the actual driving test. So, this may not be good for investigating the riding behaviour, and the behaviour may be different compared with usual riding behaviour when they are driving during the experiment. However, a riding simulator experiment is necessary to control various risk scenarios and many experimental variables. Also, the frequent accident pattern was considered for investigating the riding behaviours to quantify the riding performance, but more accident patterns should be considered in the riding simulator experiments to increase the accuracy of the quantification method. Because of the riding behaviour and factors correlated with accident may be different from accident patterns (Staubach, M., 2009; Aust, M. L. et al., 2012). Therefore, many cases of accident patterns have to carry out to increase the accuracy of data which is investigated at the riding simulator experiment in the laboratory such as the development of dynamic riding simulator.

In the quantification method, three different riding behaviour, which are brake reaction time, average deceleration, and time to collision, were investigated and quantified as a riding performance which can be represented by a single index. These variables of riding behaviours were quantified just before the risky situation occurred and after the event. 
It was used to quantify the driver's readiness or preparation for collision avoidance. Therefore, our proposed model does not quantify the driving performance during normal driving, but only quantifies the preparedness of the driver for collision avoidance. Therefore, to quantify normal operation in the absence of the risky situations as a performance amount, it would be possible to apply it to our quantification method using variables such as the amount of variation in steering, lane deviation, and traffic accident violation (Cai, H. et al., 2007; Cai, H., and Lin, Y., 2011).

\subsection{Modelling and quantifying of riding performance and collision probability using logistic regression analysis}

As mentioned in section 4.1, a logit model to predict collision probability was constructed from driver's riding behaviour under three accident patterns. Therefore, the proposed model can predict the risk of drivers from the driver's riding behaviour when similar patterns are used in modelling. But it may not be appropriate to use the proposed model to predict collision probability under other accident patterns because of the driver's riding behaviour can change in different accident patterns (Staubach, M., 2009; Aust, M. L. et al., 2012). Of course, it is considered to be the best method to generate and use each model for every accident pattern, but it is challenging to create models corresponding to all accident patterns in reality. Therefore, it is considered necessary to develop a model that can be used universally and high accuracy. In the future, it is considered that many experiments on various accident patterns are needed to generate a good model. In this paper, logistic regression analysis was performed by creating one independent variable called riding performance based on three variables consisting of, RT, AD, and TTC. The reason to consider this method is to suggest an evaluation method of riding performance to quantify the various variables to one index as the preparation for collision avoidance intuitively. To avoid the problem of multicollinearity by the correlation of each independent variable when performing the logistic regression analysis, we performed the regression analysis using only three variables: risk perception (RT), avoidance action (AD), and avoidance or collision (TTC). Variables Inflation Factor (VIF) was tested to determine whether there was a problem with multicollinearity before the logistic regression analysis. Regression analysis was performed based on the assumption that there were no problems of multicollinearity in all three variables. Although it is possible to increase the fitness of the model by using several variables, it is better to generate a model with minimum independent variables because the fitness can be increased by over-fitting. Therefore, it is necessary to consider what independent variables should be used to generate the best model. The logistic regression analysis was performed using the dataset with 64 test subjects (about 500 data for each riding behaviour), and the logit model was suggested. It was confirmed that the model was properly fitted to the investigated riding behaviours by confirming the McFadden $\mathrm{R}^{2}$ and AIC. However, since the model was suggested using the relatively small group of test subjects and data to make model for vehicle safety systems, it is necessary to consider the accuracy of the model for applying the model directly to design safety systems. Also, the test subjects in this study were limited to those who have a motorcycle license that can drive over the $250 \mathrm{cc}$ motorcycle, not a novice rider, and riding motorcycle frequently. Therefore, the model may make inappropriate decisions when it encounters behaviours other than those of the investigated test subjects.

Finally, we proposed a concept of setting method to decide the threshold of ADAS. Many ADAS have a specific threshold to present the alarm or warning, and this is decided based on the experiment or mathematics calculation. ADAS may improve the safety level by setting the variable threshold timing considering the personal characteristics of the driver's performance. But almost all the systems have just a single threshold value and riders/drivers driving performance is not considered. If the drivers have a high driving performance, the drivers may not need ADAS, and the drivers may think that the support by ADAS is too annoying. If the drivers have low driving performance, the drivers might think that support by ADAS is too unreliable. So, we suggest a method for setting the threshold which has variable timing considering riders/drivers driving performance for personalization of the system. Figure 7 (a) shows the result of the logit model, and it also shows the concept of the threshold setting method using the logit model for collision prediction based on riding performance. In our model, we clarified the point with a significant increase in collision probability and divided into three collision probability levels. In the model, we defined the low collision probability meaning $0 \%$ collision probability and riders with this collision probability level, have the perfect preparation for collision avoidance because of the high riding performance of the riders. The medium collision probability is $0.1 \%$ to $50 \%$ collision probability, and the riders have a moderate preparation for collision avoidance. And high collision probability is $50.1 \%$ to $100 \%$ collision probability, and the riders have a low preparation for collision avoidance, and it is a hazardous situation for the riders. However, as noted in section 3.2, the model to predict 
the probability of collision in accordance with the riding performance may differ according to the rider's riding styles, the ADAS needs to customize its control timing depending on the riding style of the riders. Therefore, we will explain how to apply our model to Forward Collision Warning System (FCWS), which is one of the ADAS, for setting the threshold of presentation timing according to the individual characteristics of the riders. The FCWS is one of the well-known systems of ADAS, and the primary function of FCWS is the warning of a forward object or vehicle that may collide with an ego vehicle. The FCWS has variable threshold timing of collision warning which is calculated using Stopping Distance Algorithm (SDA) or TTC, but many systems assume the brake reaction time of the driver of ego vehicle as 0.5 to 2 seconds to calculate the stopping distance (Abe, G., and Richardson, J., 2005a, 2006b). Of course, for a typical driver, it was clarified that the brake reaction time is between 0.2 and 2.5 seconds (Taoka, G. T., 1989) but using the same brake reaction time to calculate the stopping distance, is not suitable for all risky situations and all drivers. Therefore, many studies have been conducted on the safety of drivers at variable thresholds of FCWS (Abe, G., and Richardson, J., 2005a, 2006b). We demonstrated how to apply FCWS using our quantification methods and models, as shown in Fig. 7 (b). First, the system periodically stores the rider's riding behaviour and quantifies it as a riding performance. Accordingly, a model for predicting a collision is created/updated periodically. The generated model quantifies the riding performance and current collision probability in real time and changes the warning timing of FCWS according to the preparation level when the system issues the warning. For example, if the rider has a high riding performance and perfect preparation for collision avoidance, the system warns at the timing calculated by SDA. However, if the rider has a moderate riding performance and moderate preparation for collision avoidance, the system warns faster than the timing calculated in SDA to compensate for the low performance. Also, if the riding performance of the rider is too low and the collision probability is very high, it is possible to improve the safety of the rider with a warning message to stop driving immediately, and not just for the frontal collision warning. Through our method, we think that we can construct a driver personalization system by setting a variable threshold based on the performance of the driver.

\section{Conclusions}

In this study, we proposed a quantification method for the riding performance and the logit model, which can predict the collision probability based on quantified riding performance. Therefore, the conclusions are as follows:

(1) Propose a riding performance quantification method

For quantifying the riding performance, we investigated the riding behaviour when riders are in risky situations using the riding simulator. The weight value was used with generalized riding behaviour to find out which behaviour has more effect on collision and the value of overall riding performance. We confirmed $\mathrm{TTC}_{\min }$ has a negative effect on collision (The collision probability will increase as $\mathrm{TTC}_{\min }$ decreases, Pearson correlation: $-0.476, \mathrm{p}<0.001$ ) and influences collision more than any other behaviour. The brake reaction time has a positive effect on collision (The collision probability will increase as brake reaction time increases, Pearson correlation: $0.313, \mathrm{p}<0.001)$ and has a second strongest effect on collision. The average deceleration has a negative effect on collision (The collision probability will increase as average deceleration decreases, Pearson correlation: $-0.070, \mathrm{p}=0.116$ ) and has a weakness effect on collision than other parameters. The weight value was considered as a ratio of correlation coefficients of parameters.

(2) Propose a mathematical model to predict the collision probability based on the riding performance of two-wheeled riders using logistic regression analysis

The model to predict the collision for setting the threshold which can consider the riding performance was suggested using logistic regression analysis. The model can explain the collision occurrence and quantified riding performance, and McFadden $\mathrm{R}^{2}$ was 0.632 and $\mathrm{AUC}$ was 0.96 . In the model, the regression coefficient of the riding performance was -33.722 ( $\mathrm{p}<0.001$ ), and the intercept was $6.534(\mathrm{p}<0.001)$. To confirm the difference in riding performance due to their riding style, we performed a hierarchical cluster analysis based on the RSQ (Riding Style Questionnaire). There is no significant difference $(t=1.710, p=0.088)$ in riding performance of clustered group A and B. But we confirmed that the characteristic of high anxiousness and low confidence for riding enhances the riding performance (in terms of vehicle safety). Moreover, we have confirmed a difference of models for group A and B to 
predict the collision based on riding performance. We have found that riders with low anxiety and high confidence have a dramatic increase in collision probability when the riding performance is lower than a certain level. Through the model, we suggested a concept of the threshold setting for ADAS using the logit model based on collision probability and riding performance. This concept can be used to construct personalized systems by setting a variable value of threshold using the riding performance when riders are in risky situations and usual driving.

In this study, the experiments using a riding simulator were performed by three conditions of accident pattern. Therefore, the results are in a limited experiment environment, but the methodology for quantifying the riding performance and the suggested collision prediction model can be applied to other accident patterns. Based on these results, it is considered that the development of a personalized safety system by adding variables such as riding performance and riding style. The development of a personalized safety system will improve safety for driving.

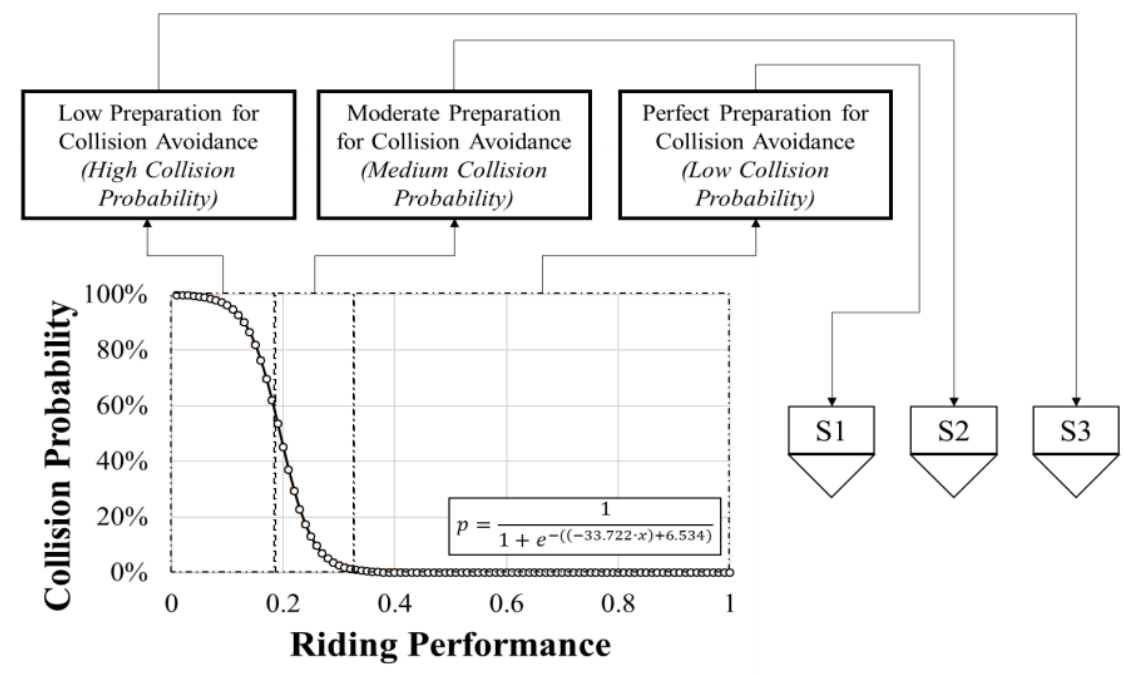

(a)

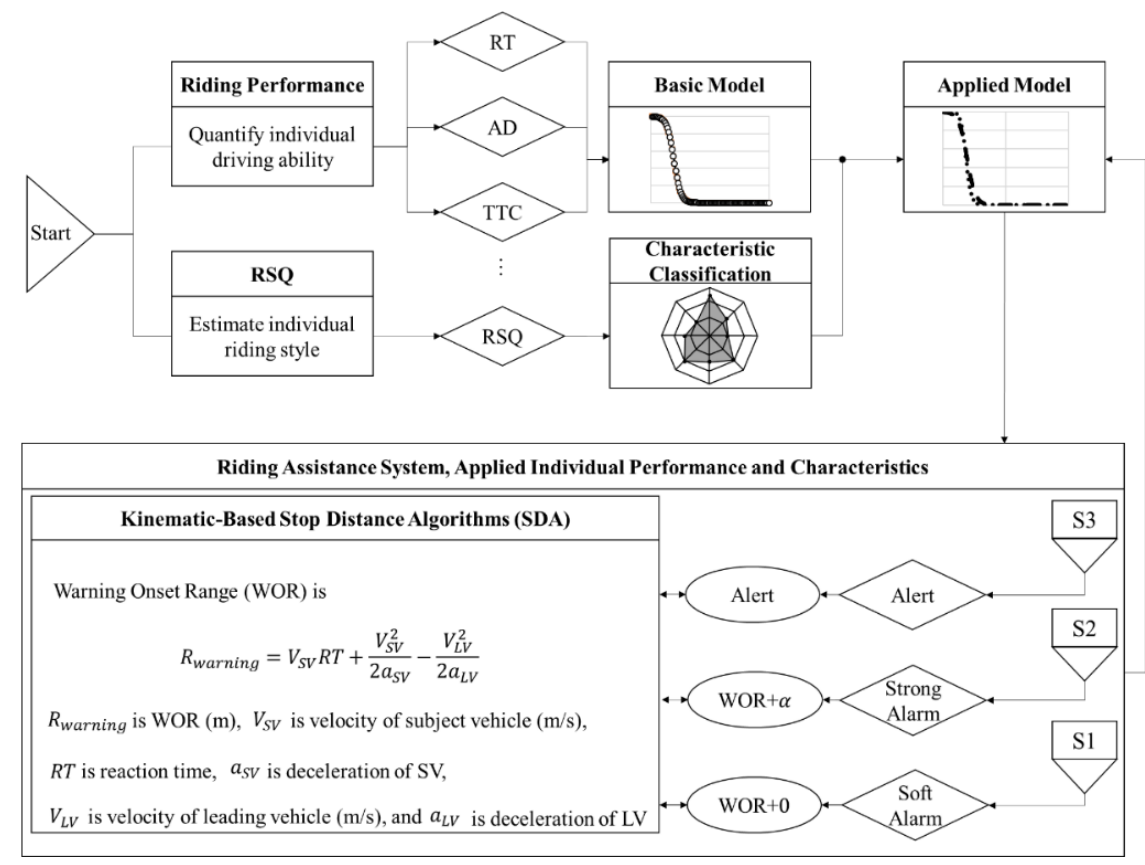

(b)

Fig. 7 A concept of threshold setting for ADASs using the logit model based on collision probability and riding performance (a) and Example for applying collision predict logit model to ADASs (b). 


\section{References}

Abe, G. and Richardson, J., The influence of alarm timing on braking response and driver trust in low speed driving, Safety Science, Vol.43, No.9 (2005), pp.639-654.

Abe, G. and Richardson, J., Alarm timing, trust and driver expectation for forward collision warning systems, Applied ergonomics, Vol.37, No. 5 (2006), pp.577-586.

Aust, M. L., Fagerlind, H. and Sagberg, F., Fatal intersection crashes in Norway: Patterns in contributing factors and data collection challenges, Accident Analysis and Prevention, Vol.45 (2012), pp.782-791.

Beede, K. E. and Kass, S. J., Engrossed in conversation: The impact of cell phones on simulated driving performance, Accident Analysis and Prevention, Vol.38 No.2 (2006), pp.415-421.

Bekiaris, E. D., Spadoni, A. and Nikolaou, S. I, SAFERIDER Project: new safety and comfort in Powered Two Wheelers, In 2009 2nd Conference on Human System Interactions (2009), pp.600-602.

Bella, F. and Russo, R., A collision warning system for rear-end collision: a driving simulator study, Procedia-social and behavioral sciences, Vol. 20(2011), pp.676-686.

Cai, H. and Lin, Y., Modeling of operators' emotion and task performance in a virtual driving environment, International Journal of Human-Computer Studies, Vol.69, No.9 (2011), pp.571-586.

Cai, H., Lin, Y. and Mourant, R. R., Study on driver emotion in driver-vehicle-environment systems using multiple networked driving simulators, In Proceedings of the Driving Simulation Conference North America (DSC'07) (2007).

Charlton, S. G., Driving while conversing: Cell phones that distract and passengers who react, Accident Analysis and Prevention, Vol.41, No.1 (2009), pp.160-173.

Chiyoda, S., Yoshimoto, K., Kawasaki, D., Murakami, Y. and Sugimoto, T., Development of a motorcycle simulator using parallel manipulator and head mounted display, In The Proceedings of the International Conference on Motion and Vibration Control 6.1 (2002), pp.599-602.

Consiglio, W., Driscoll, P., Witte, M. and Berg, W. P., Effect of cellular telephone conversations and other potential interference on reaction time in a braking response, Accident Analysis and Prevention, Vol.35, No.4 (2003), pp.495-500.

Cossalter, V., Lot, R. and Massaro, M., The chatter of racing motorcycles, Vehicle System Dynamics, Vol.46, No.4 (2008), pp.339-353.

Cossalter, V., Lot, R., Maso, M., Massaro, M. and Sartori, R., A motorcycle riding simulator for the improvement of the rider safety (2008).

Cox, D. R., The regression analysis of binary sequences, Journal of the Royal Statistical Society: Series B (Methodological), Vol.20, No.2 (1958), pp.215-232.

Creaser, J. I., Ward, N. J., Rakauskas, M. E., Shankwitz, C. and Boer, E. R., Effects of alcohol impairment on motorcycle riding skills, Accident Analysis and Prevention, Vol.41, No.5 (2009), pp.906-913.

Davoodi, S. R., Hamid, H., Pazhouhanfar, M. and Muttart, J. W., Motorcyclist perception response time in stopping sight distance situations, Safety science, Vol.50, No.3 (2012), pp.371-377.

Di Stasi, L. L., Contreras, D., Cándido, A., Cañas, J. J. and Catena, A., Behavioral and eye-movement measures to track improvements in driving skills of vulnerable road users: First-time motorcycle riders, Transportation research part F: traffic psychology and behaviour, Vol.14, No.1 (2011), pp.26-35.

Doi, A., Butsuen, T., Niibe, T., Takagi, T., Yamamoto, Y. and Seni, H., Development of a rear-end collision avoidance system with automatic brake control, Jsae Review, Vol.15, No.4 (1994), pp.335-340.

Fujita, Y., Akuzawa, K. and Sato, M., Radar brake system, Jsae Review, Vol.1, No.16 (1995), pp.113.

Gifford, J. L., ICT and road transportation safety in the United States: a case of “American exceptionalism”, IATSS research, Vol. 34, No.1 (2010), pp.1-8.

Green, M., "How long does it take to stop?" Methodological analysis of driver perception-brake times, Transportation human factors, Vo.2, No.3 (2000), pp.195-216.

Green, M. D., Orlov, N. L. and Murphy, R. W., Toward a phylogeny of the kukri snakes, genus Oligodon, Asian Herpetological Research, Vo.1, No.1 (2010), pp.1-21.

Hayakawa, H., Fischbeck, P. S. and Fischhoff, B., Traffic accident statistics and risk perceptions in Japan and the 
United States, Accident Analysis and Prevention, Vo.32, No.6 (2000), pp.827-835.

Hirst, S. and Graham, R., The format and presentation of collision warnings, Ergonomics and safety of intelligent driver interfaces (1997), pp.203-219.

Institute for Traffic Accident Research and Data Analysis, Accident of motorcycle accident, ITARDA Information, No. 52 (2004) (in Japanese).

Institute for Traffic Accident Research and Data Analysis, Features of motorcycle accident: Beware of "overlooks”!, ITARDA Information, No. 91 (2011) (in Japanese).

Institute for Traffic Accident Research and Data Analysis, Serious T-born accidents of motorcycle and low-speed driving automobile, ITARDA Information, No. 105 (2014) (in Japanese).

Institute for Traffic Accident Research and Data Analysis, Traffic Statistics (2017) (in Japanese).

Ishibashi, M., Okuwa, M., Doi, S. and Akamatsu, M., Characterizing Indices of Driver's Workload Sensitivity and their Relevance to Route Choice Preferences, Society of Automotive Engineers of Japan, Vol.39, No.5 (2008), pp.169-174 (in Japanese).

Ishibashi, M., Okuwa, M., Doi, S. and Akamatsu, M., Characterizing Indices of Driving Style and their Relevance to Car Following Behavior, TRANSACTIONS-SOCIETY OF AUTOMOTIVE ENGINEERS OF JAPAN, Vol.39, No.1 (2008), pp.121 (in Japanese).

Jurecki, R. S., Jaśkiewicz, M., Guzek, M., Lozia, Z. and Zdanowicz, P., Driver's reaction time under emergency braking a car-research in a driving simulator, Eksploatacja i Niezawodność, Vol.14 (2012), pp.295-301.

Kuraishi, S., Development of the "Uchida-Kuraepelin Psychodiagnostic Test" in Japan., Psychologia, Vol.1 (2000), pp.104-109.

Lee, H. C., Cameron, D. and Lee, A. H., Assessing the driving performance of older adult drivers: on-road versus simulated driving, Accident Analysis and Prevention, Vol.35, No.5 (2003), pp.797-803.

Lee, J., Ozaki, I., Kishino, S., Suzuki, K. and Nakajima, M., Human-Machine Interface System for Motorcyclists: Quantification of Total Reliability as Human-Machine System, In International Conference on Applied Human Factors and Ergonomics (2018), pp.604-614.

Lee, K. and Peng, H., Evaluation of automotive forward collision warning and collision avoidance algorithms, Vehicle system dynamics, Vol.43, No.10 (2005), pp.735-751.

Liu, H., Wei, H., Zuo, T., Li, Z. and Yang, Y. J., Fine-tuning ADAS algorithm parameters for optimizing traffic safety and mobility in connected vehicle environment, Transportation research part C: emerging technologies, Vol.76 (2017), pp.132-149.

Mayberry, J. C., Pearson, T. E., Wiger, K. J., Diggs, B. S. and Mullins, R. J., Equestrian injury prevention efforts need more attention to novice riders, Journal of Trauma and Acute Care Surgery, Vol.62, No.3 (2007), pp.735-739.

Mayhew, D. R., Simpson, H. M., Wood, K. M., Lonero, L., Clinton, K. M. and Johnson, A. G., On-road and simulated driving: Concurrent and discriminant validation, Journal of safety research, Vol.42, No.4 (2011), pp.267-275.

Ministry of Land, Infrastructure, Transport and Tourism, Guidelines for communication assisted driving support system (2001).

Mochizuki, M., Kubotani, H., Nii, Y. and Suzuki, K., Evaluation of the effectiveness of collision-prevention support information at a blind intersection and analysis concerning the acceptable reliability of the sensors, Transactions of Society of Automobile Engineers of Japan, Vol.44, No.2 (2013) (in Japanese).

Mochizuki, M., Nii, Y., Suzuki, K. and Yamada, K., Evaluation of the Distraction Reduction by Driving Support Systems in a Collision at Intersection Based on the Time Series Reliability Model, Transactions of The Society of Instrument and Control Engineers, Vol.49 (2013), pp.1074-1081 (in Japanese).

Nehaoua, L., Hima, S., Arioui, H., Seguy, N. and Espié, S., Design and modeling of a new motorcycle riding simulator, In 2007 American Control Conference (2007), pp.176-181.

Ohlhauser, A. D., Milloy, S. and Caird, J. K., Driver responses to motorcycle and lead vehicle braking events: The effects of motorcycling experience and novice versus experienced drivers, Transportation research part F: traffic psychology and behaviour, Vol.14, No.6 (2011), pp.472-483.

Okuda, R., Kajiwara, Y. and Terashima, K., A survey of technical trend of ADAS and autonomous driving, In Technical Papers of 2014 International Symposium on VLSI Design, Automation and Test (2014), pp.1-4.

Philip, P., Sagaspe, P., Moore, N., Taillard, J., Charles, A., Guilleminault, C. and Bioulac, B., Fatigue, sleep restriction and driving performance, Accident Analysis and Prevention, Vol.37, No.3 (2005), pp.473-478. 
Staubach, M., Factors correlated with traffic accidents as a basis for evaluating Advanced Driver Assistance Systems, Accident Analysis and Prevention, Vol.41, No.5 (2009), pp.1025-1033.

Suzuki, K., Mochizuki, M., Micchi, Y. and Yamada, K., Methodologies for evaluating the effectiveness of collision avoidance assistance system, Transactions of the JSME (in Japanese), Vol.82, No.835 (2016), DOI:10.1299/transjsme.15-00565.

Suzuki, K., Yamaguchi, H. and Oguri, T., Designing of brake timing of autonomous emergency braking system to avoid collision to pedestrians, Mechanical Engineering Journal, Vol.4, No.5 (2017), DOI:10.1299/mej.17-00215.

Taoka, G. T., Brake reaction times of unalerted drivers, ITE journal, Vol.59, No.3 (1989), pp.19-21.

Vahidi, A. and Eskandarian, A., Research advances in intelligent collision avoidance and adaptive cruise control, IEEE transactions on intelligent transportation systems, Vol. 4, No.3 (2003), pp.143-153.

Van Der Hulst, M. and Geurts, S., Associations between overtime and psychological health in high and low reward jobs, Work and Stress, Vol.15, No.3 (2001), pp.227-240.

Van Der Hulst, M., Meijman, T. and Rothengatter, T., Maintaining task set under fatigue: a study of time-on-task effects in simulated driving, Transportation research part F: traffic psychology and behaviour, Vol.4, No.2 (2001), pp.103-118.

Wang, X., Chen, M., Zhu, M. and Tremont, P., Development of a kinematic-based forward collision warning algorithm using an advanced driving simulator, IEEE Transactions on Intelligent Transportation Systems, Vol.17, No.9 (2016), pp.2583-2591.

Weir, D. H., Application of a driving simulator to the development of in-vehicle human-machine-interfaces, IATSS research, Vol.34, No.1 (2010), pp.16-21.

Wilson, T. B., Butler, W., McGehee, D. V. and Dingus, T. A., Forward-looking collision warning system performance guidelines, SAE transactions (1997), pp.701-725. 